\title{
Tratamento endodôntico mecanizado em dentição decídua
}

\author{
Mechanized endodontic treatment in deciduous dentition \\ Tratamiento endodóntico mecanizado en dentición decidua
}

\section{Resumo}

A instrumentação mecanizada em dentes permanentes já é uma prática consolidada e muito utilizada na odontologia atual. Diversos estudos apresentam vantagens consideráveis, quando comparada com a técnica de instrumentação manual, entre elas pode-se considerar a redução de tempo cirúrgico e o menor desconforto ao paciente durante e após o procedimento. Apesar do custo ser consideravelmente maior quando comparado ao tratamento realizado com instrumentação manual, este é justificável. Entretanto, a endodontia mecanizada para tratamentos endodônticos em odontopediatria ainda é questionável. O objetivo desta revisão de literatura foi identificar vantagens e desvantagens do procedimento endodôntico mecanizado em odontopediatria. Para tanto, realizamos levantamento bibliográfico, considerando publicações do período de 2015 a 2020 nas plataformas PubMed, Google Scholar, Scielo, Springer e Cochrane. Foram selecionados 17 artigos, dentre eles, estudos realizados in vitro, revisões sistemáticas e estudos randomizados e duas referências bibliográficas, o Manual de Odontopediatria (Guedes-Pinto, 2012) e o manual "Diretrizes para Procedimentos Clínicos em Odontopediatria - ABO”, lançado em 2020. Após a triagem dos materiais, 8 artigos, o Manual de Odontopediatria (Guedes-Pinto) e o Manual da ABO foram incluídos nesta revisão. Concluiu-se que, apesar dos significativos benefícios apresentados na instrumentação mecanizada em dentes permanentes, não foram observadas vantagens da técnica quando aplicada em odontopediatria, injustificando o alto investimento.

Palavras-chave: Endodontia; Odontopediatria; Instrumentação mecanizada; Dentes decíduos.

\begin{abstract}
Mechanized instrumentation in permanent teeth is already a well-established and widely used practice in dentistry today. Several studies show considerable advantages when compared to the manual instrumentation technique, among them one can consider the reduction of surgical time and less discomfort to the patient during and after the procedure. Although the cost is considerably higher when compared to the treatment performed with manual instrumentation, this is justifiable. However, mechanized endodontics for endodontic treatments in pediatric dentistry is still questionable. The objective of this literature review was to identify advantages and disadvantages of the mechanized endodontic procedure in pediatric dentistry. Therefore, we carried out a bibliographic survey, considering publications from 2015 to 2020 on the PubMed, Google Scholar, Scielo, Springer and Cochrane platforms. Seventeen articles were selected, including in vitro studies, systematic reviews, and randomized studies and two bibliographic references, the Pediatric Dentistry Manual (Guedes-Pinto, 2012) and the manual "Guidelines for Clinical Procedures in Pediatric Dentistry ABO", released in 2020. After screening the materials, 8 articles, the Pediatric Dentistry Manual (Guedes-Pinto) and the ABO Manual were included in this review. It was concluded that, despite the significant benefits presented by mechanized instrumentation in permanent teeth, no advantages of the technique were observed when applied in pediatric dentistry, unjustifying the high investment.
\end{abstract}

Keywords: Endodontics; Pediatric dentistry; Mechanized instrumentation; Deciduous teeth; Root canal.

\section{Resumen}

La instrumentación mecanizada en dientes permanentes ya es una práctica bien establecida y ampliamente utilizada en odontología en la actualidad. Varios estudios muestran ventajas considerables en comparación con la técnica de 
instrumentación manual, entre ellas se puede considerar la reducción del tiempo quirúrgico y menos molestias para el paciente durante y después del procedimiento. Si bien el costo es considerablemente mayor en comparación con el tratamiento realizado con instrumentación manual, esto es justificable. Sin embargo, la endodoncia mecanizada para tratamientos de endodoncia en odontopediatría sigue siendo cuestionable. El objetivo de esta revisión de la literatura fue identificar las ventajas y desventajas del procedimiento de endodoncia mecanizada en odontopediatría. Por ello, realizamos un relevamiento bibliográfico, considerando publicaciones de 2015 a 2020 en las plataformas PubMed, Google Scholar, Scielo, Springer y Cochrane. Se seleccionaron diecisiete artículos, entre estudios in vitro, revisiones sistemáticas y estudios aleatorizados, y dos referencias bibliográficas, el Manual de Odontopediatría (Guedes-Pinto, 2012) y el manual “Guías de Procedimientos Clínicos en Odontopediatría - ABO”, publicado en 2020. Después de la selección de los materiales, se incluyeron en esta revisión 8 artículos, el Manual de Odontopediatría (Guedes-Pinto) y el Manual ABO. Se concluyó que, a pesar de los importantes beneficios que presenta la instrumentación mecanizada en dientes permanentes, no se observaron ventajas de la técnica cuando se aplica en odontopediatría, injustificando la alta inversión.

Palabras clave: Endodoncia; Odontología pediatrica; Instrumentación mecanizada; Dientes caducifolios.

\section{Introdução}

A dentição decídua desenvolve um papel muito importante no desenvolvimento da criança. Esses são responsáveis pela mastigação, fonação e estética, além disso, a dentição decídua exerce a função de um mantenedor de espaço, cuja função é permitir que o dente permanente irrompa na posição e cronologia desejadas (Katge et al. 2016; Associação Brasileira de Odontopediatria (ABO, 2020); \& Hecksher et al. 2018).

Os primeiros dentes decíduos irrompem por volta dos 6 meses de idade, embora exista uma variabilidade neste período e, entre 23 e 33 meses a criança tem a dentição decídua completa, permanecendo na cavidade bucal até aproximadamente os 12 anos de idade (Associação Brasileira de Odontopediatria (ABO, 2020). Ainda de acordo com a Associação Brasileira de Odontopediatria (ABO, 2020), é nesta fase de desenvolvimento da criança que a perda precoce pode causar inúmeros danos à saúde bucal, dentre eles problemas no desenvolvimento facial e do arco dentário corroborando com o mal posicionamento da dentição permanente, ocasionando problemas de oclusão, fonação, mastigação e impactos psicológicos por questões relacionadas a estética (Katge et al. 2016 \& Silva et al., 2015). Para Guedes \& Moura, 2012, um dos papéis fundamentais da odontopediatria é a preservação do órgão dental decíduo na cavidade oral até o momento da sua esfoliação fisiológica e o irrompimento dos dentes permanentes.

De acordo com Katge et al. 2016, os dentes decíduos servem como guia para o irrompimento dos dentes permanentes, além disso, previnem hábitos deletérios. Apesar dos grandes esforços e avanços dos programas de saúde que visam a orientação e conscientização dos pais acerca da saúde bucal das crianças, ainda é comum, principalmente em populações com problemas socioeconômicos, crianças com dentes decíduos com grandes destruições coronárias (ICDAS 5 e 6), tendo como fator etiológico extensas lesões cariosas, principalmente na faixa etária de 4 a 6 anos. Os traumas dentais também estão muito presentes nesta mesma faixa etária levando muitas vezes à necessidade de tratamentos endodônticos em crianças.

O tratamento endodôntico em dentes decíduos sempre foi realizado com limas manuais, entretanto atualmente, tem crescido muito o uso de instrumentação com rotatórios em endodontia. Na dentição permanente, esta é uma prática comum, cujas vantagens já foram demonstradas e são bastante significativas, dentre elas, a melhor modelagem dos canais, redução do tempo clínico e da dor pós-operatória. Desta forma, o objetivo deste trabalho foi realizar uma revisão de literatura para avaliar as possíveis vantagens e desvantagens de se realizar o tratamento endodôntico com instrumentação mecanizada em dentes decíduos.

\section{Metodologia}

Foram utilizados métodos de pesquisa em banco de dados como Scielo, Springer, Cochrane, Google Scholar e PubMed, com base no título inicial, utilizando das palavras-chave em português (Endodontia; Odontopediatria; Instrumentação 
Mecanizada; Dentes decíduos) e inglês (Endodontics; Pediatric Dentistry; Mechanized Instrumentation; Deciduous teeth, Root canal). Como critério de exclusão, consideramos o ano de publicação, na primeira seleção, consideramos o período de 2018 a 2020, como a amostragem não foi significativa, estendemos o período para 2011 a 2020. Inicialmente foram selecionados 32 trabalhos, sendo estudos realizados em in vitro, revisões sistemáticas e estudos randomizados em que diferentes tipos de técnicas de instrumentação rotatória foram comparados com técnicas manuais para tratamento de canal radicular em dentes decíduos. Consideramos ainda, duas referências bibliográficas, o Manual de Odontopediatria - Guedes-Pinto (2012) e o Manual de "Diretrizes para Procedimentos Clínicos em Odontopediatria - ABO", lançada no ano de 2020. Por fim, dentre os artigos pesquisados, selecionamos 17 estudos para realizar esta revisão. A pesquisa também foi realizada em revistas e anuários, os conteúdos utilizados foram encontrados na revista RevOdonto ${ }^{\circledR}$ e Anuário ABO.

\section{Revisão de Literatura e Discussão}

Segundo Hülsmann, 2018, o objetivo da instrumentação é preparar o espaço do canal para facilitar a desinfecção por irrigantes e medicamentos. As limas para instrumentação manual são classificadas em usinadas ou torcidas. As limas torcidas apresentam menor potencial cortante e são conhecidas pelos tipos K e Flexofile. São instrumentos bastante flexíveis, em especial a Flexofile que está indicada para canais mais curvos. As limas tipo K são as mais utilizada no dia a dia do endodontista é o instrumental mais indicada para uso em odontopediatria.

As limas K estão disponíveis nos tamanhos 21, 25 e 31mm, com exceção as Flexofile, que estão disponíveis apenas nos tamanhos 21 e $25 \mathrm{~mm}$. As limas Hedströem são usinadas, estão disponíveis no comprimento de $17 \mathrm{~mm}$, medida considerada ideal para odontopediatria, no entanto, possuem alto poder de corte e por este motivo estão contraindicadas para uso em tratamentos endodônticos em dentes decíduos. As limas K, Flexofile e Hedströem são produzidas em aço inoxidável e quanto ao diâmetro, são classificadas em série especial (\#6, 8 e 10), primeira série (\#15 a 40), segunda série (\#45 a 80) e terceira série (raramente utilizadas \#80 a 140).

Figura 1: Especificações do instrumento manual.

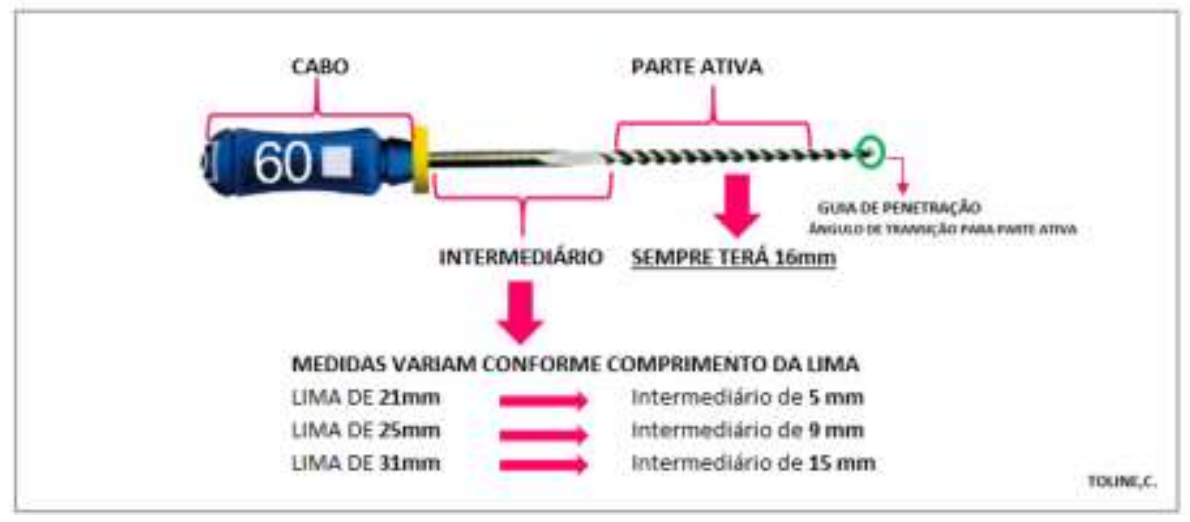

Fonte: Imagem de internet. Esquema de identificação das partes do instrumental: Material elaborado pelos autores.

Os dentes decíduos apresentam canais radiculares mais delgados, desta forma, a instrumentação manual deve acontecer com limas de no máximo \#30, ou seja, 0,30mm de diâmetro na ponta do instrumento. Todas as limas manuais possuem $16 \mathrm{~mm}$ de ponta ativa.

Os instrumentos mecanizados estão classificados em dois grupos: Rotatórios e Reciprocantes, ambos instrumentais são produzidos em ligas de NiTi (liga de Níquel e Titânio), que conferem maior flexibilidade ao instrumento. 
Figura 2: Instrumento rotatório inserido em canal curvo.

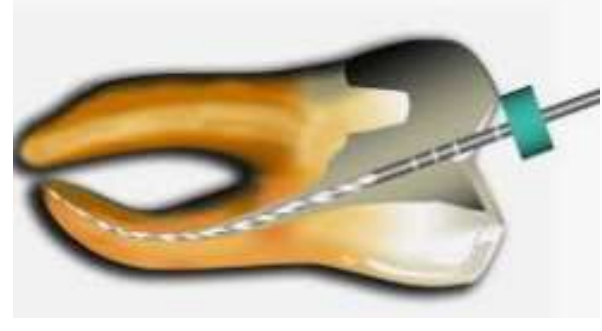

Fonte: Alipratica - PT - Imagem de internet.

A diferença básica entre os sistemas refere-se ao sentido de rotação, sendo:

Rotatório: A lâmina gira em sentido horário (para direita). A rotação é realizada em um único sentido;

Reciprocante: Neste sistema, a lâmina possui rotação em $150^{\circ}$ em sentido anti-horário (para a esquerda) e $30^{\circ}$ em sentido horário, minimizando a resistência e possível travamento da lima no interior do canal. As marcas comerciais mais utilizadas são WaveOne Gold ${ }^{\circledR}$, fabricada pela empresa Dentsply Sirona e Reciproc ${ }^{\circledR}$ fabricada pela VDW, as demais marcas seguem os mesmos padrões. Para ambos os sistemas, preconiza-se realizar o máximo de três inserções no interior do canal, após irrigação e exploração manual com lima de memória (limas de série especial e primeira série 8,10 ou 15). De acordo com Prabhakar et al. 2016, os sistemas reciprocantes de lima única são mais rápidos e minimizam os erros de procedimento e, portanto, podem ser recomendados para moldar os canais radiculares dos dentes decíduos. Embora os instrumentos sejam mais flexíveis, reduzindo consideravelmente o risco de fratura por fadiga, recomenda-se que não sejam utilizadas por mais de três vezes, ou seja, três atendimentos clínicos. Os fabricantes preconizam o uso único, no entanto, na realidade brasileira com os custos consideravelmente elevados, esse descarte se tornou incomum. De acordo com os estudos já realizados com os materiais em NiTi (níquel-titânio) utilizados na fabricação destas limas, a deformação acontece gradualmente e a partir da terceira passagem pelo ciclo de esterilização, os riscos de fratura aumentam e o corte se torna ineficaz e, contraindicando a reutilização, portanto deve ser descartada.

Figura 3: Instrumento rotatório - Pro-Taper® fabricada em Níquel Titânio.

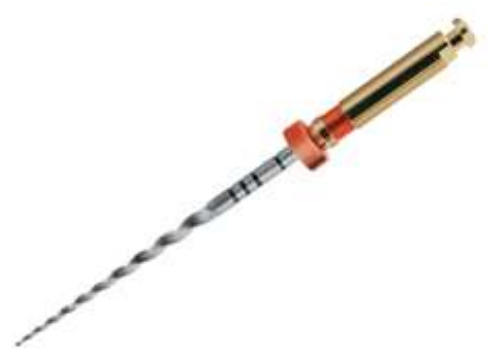

Fonte: Site do fabricante Dentsply Sirona. 
Figura 4: Instrumento reciprocante - Reciproc $®$

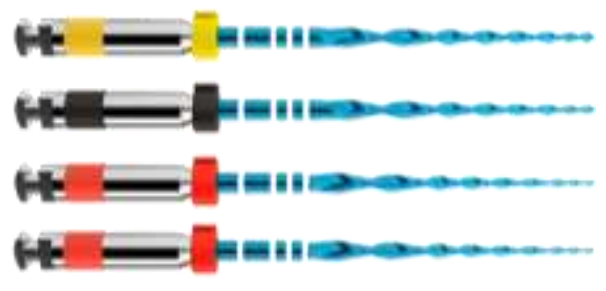

Fonte: Site VDW.

Figura 6: Equipamento Rotatório e Reciprocante.

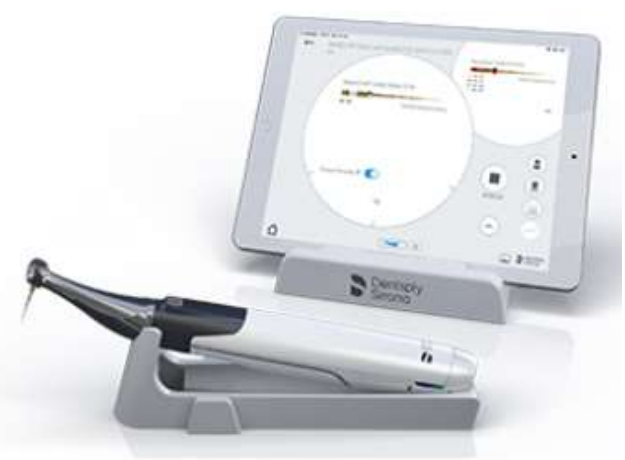

Fonte: Site Dentsply Sirona ${ }^{\circledR}$
Figura 5: Instrumento Reciprocante - Wave One® Gold.

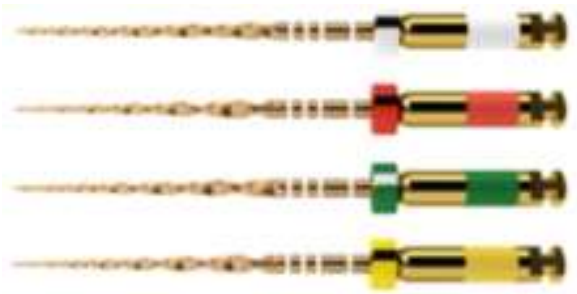

Fonte: Site Dentsply Sirona.

Figura 7: Equipamento Rotatório e Reciprocante.

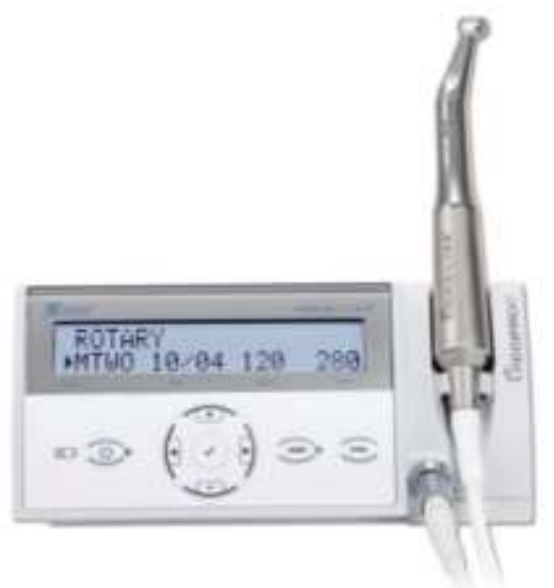

Fonte: Site VDW®

Figura 8: Instrumento reciprocante - Reciproc $®$ fabricada em Níquel Titânio.

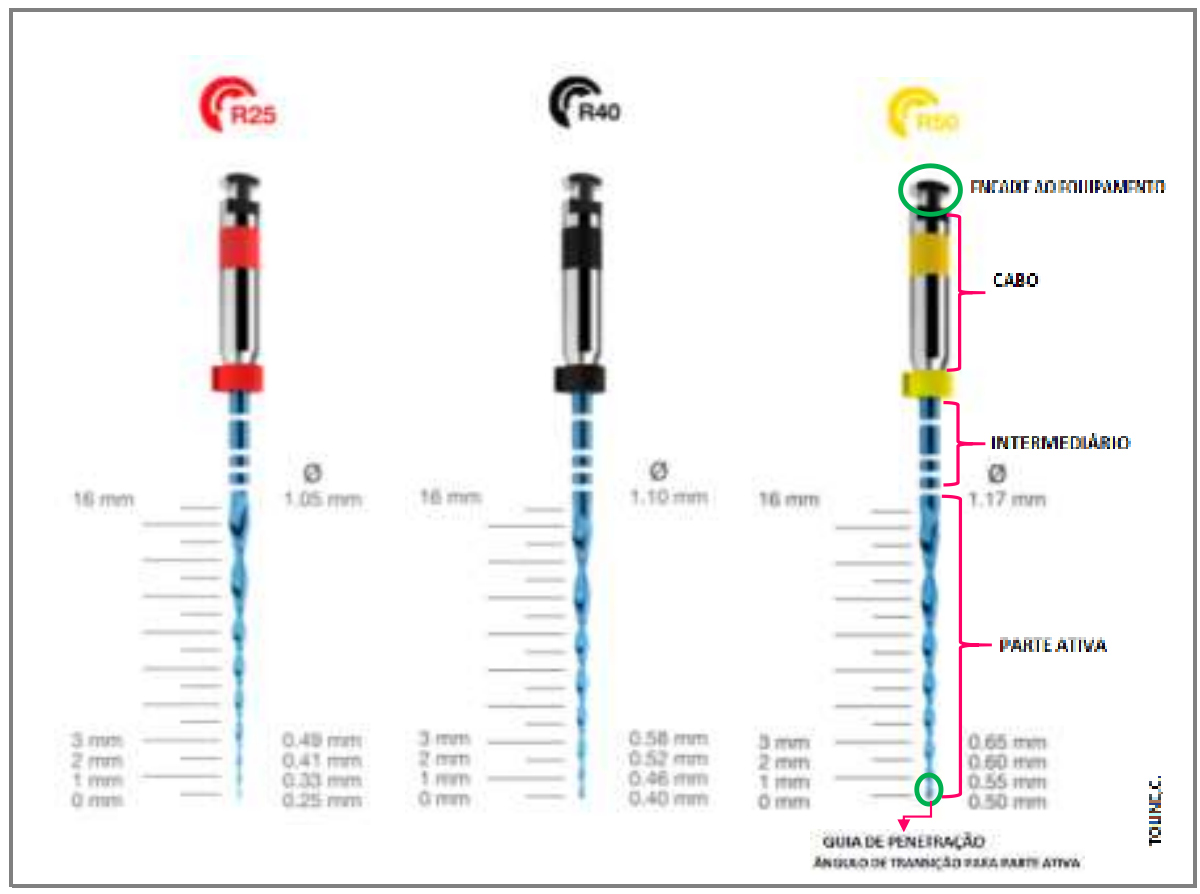

Fonte: Imagem de internet- Adaptado pela autora. 
A instrumentação mecanizada exige maior habilidade do operador e possui custo mais expressivo, aproximadamente 7 vezes mais alto quando comparado com as limas manuais. Segundo Govindaraju et al., 2017, a limitação potencial para seu uso em crianças pode ser devida à morfologia dos dentes decíduos, além das dificuldades inerentes ao atendimento infantil. De acordo com estudo comparativo realizado por Silva et al, 2015 e Manchanda et al. 2020, o tempo de execução dos procedimentos, comparando as duas técnicas (manual e mecanizadas) não foram observadas redução significativas, no entanto a instrumentação mecanizada apresentou pequena vantagem.

Quanto a eficácia da instrumentação manual e rotativa, Panchal et al. 2019, concluiu em estudo clínico randomizado que instrumentação rotativa apresenta eficiência de limpeza equivalente às limas manuais dependendo do sistema de instrumentação e das técnicas utilizadas. No entanto, o uso de limas rotativas nos dentes decíduos leva a uma melhor conformação dos canais, proporcionando melhor qualidade de tratamento em menos tempo.

Em relação a dor pós-operatória, Panchal et al. 2019, realizou um estudo clínico com 69 crianças na faixa etária de 4 a 6 anos, foram utilizadas as limas manuais K e Hedströen e limas rotativas Kedo-S, foram monitorados os intervalos de 6, 12, 24, 48 e 72 horas de pós-operatório usando a escala de Wong-Baker.

Concluiu-se que no intervalo de 6 e 12 horas, os pacientes submetidos a instrumentação rotativa apresentou menor dor em relação ao grupo cuja instrumentação foi manual. No intervalo de 24, 48 e 72 horas não foi observada nenhuma diferença significativa entre os grupos de instrumentação com limas rotativas e manual. Resultados semelhantes foram observados por Manchanda et al. 2020, no entanto, no estudo realizado não foi possível concluir que a limpeza do sistema de canais radiculares é mais eficiente quando comparada a instrumentação manual.

\section{Discussão}

A instrumentação é uma etapa importante do tratamento endodôntico e a escolha do instrumental deve ser feita com o intuito de garantir maior segurança ao operador e paciente, maior agilidade e efetividade na realização do procedimento. As condições socioeconômicas do paciente também serão um fator importante na escolha da instrumentação.

Os avanços tecnológicos impactaram positivamente na odontologia, e na endodontia, a instrumentação mecanizada se tornou forte aliada dos cirurgiões-dentistas em tratamentos na dentição permanente. Por outro lado, em odontopediatria a instrumentação manual ainda é a mais utilizada.

Os instrumentos rotatórios modelam melhor o canal radicular, no entanto, este ponto pode não ser relevante para dentes decíduos, uma vez que os canais são mais delgados e precisamos ter mais cuidado ao realizar o desgaste. No entanto para Prabhakar et al. 2016, os instrumentos rotatórios reduzem o tempo de trabalho, sendo este o mais adequado para o tratamento de dentes decíduos, pois aumenta a cooperação da criança durante o procedimento endodôntico. Porém, para a dentição decídua preconiza-se pela abertura coronária e modelagem suficientes para o bom escoamento do material obturador (pasta).

Embora, Musale et al. (2014) e Jafarzadeh et al. (2019), consideraram melhor qualidade de obturações com a técnica de instrumentação rotatória quando comparada com a manual.

$\mathrm{Na}$ prática clínica, a instrumentação manual confere maior "controle tátil” ao operador, quando consideramos o comportamento da criança, desta forma, mitigamos o risco e gravidade de possíveis acidentes. Há um consenso entre os autores estudados ao que se refere à eficiência e importância da instrumentação, seja ela manual ou rotatória. 


\section{Conclusão}

Embora o tratamento endodôntico realizado com sistema mecanizado em dentes permanentes apresente vantagens significativas, em odontopediatria o alto investimento é questionável, visto que não existem estudos clínicos bem delineados que apontem uma taxa de sucesso superior ao uso de instrumentação manual.

Apesar dos recentes avanços observados na endodontia, há poucas evidências in vivo, sobre a efetividade da instrumentação mecanizada em dentes decíduos. Desta forma, justifica-se necessidade da realização de trabalhos adicionais, acrescentando a avaliação da qualidade da obturação do SCR e as taxas de sucesso clínico do tratamento.

\section{Referências}

ABO. (2020). Diretrizes para Procedimentos Clínicos em Odontopediatria. Associação Brasileira de Odontopediatria. Santos. 2020. 19-21. 153-162.

Birck, D. R.. Miyagaki, D. C.. Corralo, D. J.. \& Tonial, F. (2016). Estudo in vitro sobre a efetividade de desinfetantes na limpeza de cones de guta-percha. RFO Revista da Faculdade Odontologia de Passo Fundo, 21, 2. 10.5335/rfo.v21i2.6103

Estrela, C. (2018). Metodologia Científica: Ciência, Ensino, Pesquisa. Editora Artes Médicas.

Guedes-Pinto A. C., Haddad A. E., Mello-Moura, A. C. V., Fraga, C. P. T., Zardetto, C. G., Raggio, D. P., Guedes-Pinto, E., Santos, E. M., Almeida, E. R. B. H., Imparato, J. C. P., Motta, L. F. G., Bönecker, M., Wanderley, M. T., Corrêa, M. S. N. P., Fonoff, R. N., Bussadori, S. K., Chedid, S. J., \& Ferreira, S. L. M. (2012). Manual de Odontopediatria. Manual de Odontopediatria. Ed. Santos.

Govindaraju, L., Jeevanandan, G., \& Subramanian, E. (2017). Clinical Evaluation of Quality of Obturation and Instrumentation Time using Two Modified Rotary File Systems with Manual Instrumentation in Primary Teeth. Journal of clinical and diagnostic research: JCDR, 11(9), ZC55-ZC58. https://doi.org/10.7860/JCDR/2017/30069.10602

Hecksher, F., Vidigal, B., Coelho, P., Otoni, D., Alvarenga, C., \& Nunes, E. (2018). Endodontic treatment in artificial deciduous teeth by manual and mechanical instrumentation: A pilot study. International journal of clinical pediatric dentistry, 11(6), 510-512. 10.5005/jp-journals-10005-1566

Hüslman, M. (2018). Notas de aula referentes a citação durante a aula do Prof. Dr. Carlos Bueno, 2020.

Jafarzadeh, M., Saatchi, M., Jafarnejadi, P., \& Gooran, M. (2019). Digital Radiographic Evaluation of the Quality of Different Root Canal Obturation Techniques in Deciduous Mandibular Molars after Preparation with Rotary Technique. Journal of dentistry (Shiraz, Iran), 20 (3), 152-158. doi.org/10.30476/DENTJODS.2019.44902

Katge F., Chimata, V. K., Poojari, M., Shetty, S., \& Rusawat, B. (2016). Comparison of cleaning efficacy and instrumentation time between rotary and manual instrumentation techniques in primary teeth: An in vitro study. International Journal of Clinical Pediatric Dentistry, 124-127. doi:10.5005/jp-journals-100051347

Manchanda, S., Sardana, D., \& Yiu, C. K. Y. (2020). A systematic review and meta-analysis of randomized clinical trials comparing rotary canal instrumentation techniques with manual instrumentation techniques in primary teeth. International Endodontic Journal, 53, 333-353. doi:10.1111/iej.13233.

Mello-Moura, A. C. V., Moura-Netto, C., Moura, A. A. M., Guedes-Pinto, A. C., \& Mendes, F. M. (2018). Tratamento endodôntico em dentes decíduos. Anuário de Odontopediatria Clínica. 154-167. http://www.fo.usp.br/pos/wp-content/uploads/2018/03/Tratamento-Endodontico-em-dentes-deciduos.pdf

Musale, P. K., \& Mujawar, S. A. (2014). Evaluation of the efficacy of rotary vs. hand files in root canal preparation of primary teeth in vitro using CBCT. European archives of paediatric dentistry: official journal of the European Academy of Paediatric Dentistry, 15(2), 113-120. doi.org/10.1007/s40368013-0072-1

Panchal, V., Jeevanandan, G., \& Erulappan, S. M. (2019). Comparison between the Effectiveness of Rotary and Manual Instrumentation in Primary Teeth: A Systematic Review. International journal of clinical pediatric dentistry, 12(4), 340-346. doi:10.5005/jp-journals-10005-1637

Panchal, V., Jeevanandan, G., \& Subramanian, E. M. G. (2019). Comparison of post-operative pain after root canal instrumentation with hand K-files, H-files and rotary Kedo-S files in primary teeth: a randomised clinical trial. European Archives of Paediatric Dentistry, 467-472. doi:10.1007/s40368-019-00429-5

Prabhakar, A. R., Yavagal, C., Dixit, K., \& Naik, S. V. (2016). Reciprocating vs Rotary Instrumentation in Pediatric Endodontics: Cone Beam Computed Tomographic Analysis of Deciduous Root Canals using Two Single-file Systems. International journal of clinical pediatric dentistry, 9(1), 45-49. 10.5005 /jpjournals-10005-1332

Preteli, H., Bezzoni, F., Faleiros, F. B. C., Dametto, F. R., \& Vaz, L.G. (2011). Comparação entre soluções irrigadoras na endodontia: clorexidina x hipoclorito de sódio. RGO Rev.Gauc.Odont. 59 (supl.1), 127-132. http://revodonto.bvsalud.org/pdf/rgo/v59s1/a18v59s1.pdf

Silva, A. M. V., Lima, M. G. S., Figueiredo, M. G. F., Santos Júnior, V. E., Pereira, J. R. D., \& Rosenblatt, A. (2015). Observação dos critérios para indicação de tratamento endodôntico em dentes decíduos na prática clínica. Odontologia. Clínico- Científica. 14, 1, 571-574. http://revodonto.bvsalud.org/pdf/occ/v14n1/a05v14n1.pdf

Siqueira Jr., J. F., Rôças, I. N., Lopes, H. P., Alves, F. R. F.. Oliveira, J. C. M., Armada, L., \& Provenzano, J.C. (2012). Princípios biológicos do tratamento endodôntico de dentes com polpa necrosada e lesão perirradicular. Revista Brasileira de Odontologia. 69, 1, 8-14. http://revodonto.bvsalud.org/pdf/rbo/v69n1/a04v69n1.pdf 
Research, Society and Development, v. 10, n. 13, e391101321345, 2021

(CC BY 4.0) | ISSN 2525-3409 | DOI: http://dx.doi.org/10.33448/rsd-v10i13.21345

Imagens de Instrumentos e Equipamentos mecanizados:

https://www.dentsplysirona.com/pt-br

https://www.vdw-dental.com/en/ 